\title{
O TOQUE E A DISTÂNCIA INTERPESSOAL ENTRE ENFERMEIROS E PACIENTES NAS CONSULTAS DE ENFERMAGEM
}

SILVA, M.J.P. da $O$ toque e a distància interpessoal entre enfermeiros e pacientes nas consultas de enfermagem. Rev. Esc. Enf. USP, v. 25, n. 3, p. 309-18, dez. 1991.

$O$ presente trabalho relata as observacoes feitas durante as consultas de enfermagem de adultos em um hospital da cidade de São Paulo, quanto ao momento $e$ local em que o enfermeiro $e$ o paciente se tocaram durante as consultas, a distância interpessoal mantida entre eles durante todo o tempo, e tece comentários sobre o significado desses sinais não verbais na interação interpessoal. Discute também os objetos utilizados pelo enfermeiro e paciente que serviram para a identificação ou definição da relação mantida.

UNITERMOS: Toque, Distância interpessoal. Comunicação não verbal.

\section{INTRODUÇĀO}

$\mathrm{Na}$ interação face a face os códigos de comunicação são audiveis e também visíveis e sensíveis. Comunicamo-nos com a linguagem verbal, ou seja, com os sons emitidos pelo aparelho fonador e com o corpo todo, inclusive com os objetos e adornos utilizados por nós.

Os elementos não-verbais de um processo comunicativo, entende-se por comunicação não-verbal todas as formas de comunicação que não envolvam as palavras expressas ${ }^{20}$, são vários e classificados diferentemente segundo os autores lidos $1,4,5,9,17,19,20,23$.

STEINBERG ${ }^{23}$ refere que a comunicação voluntária e involuntária (consciente e inconsciente) é transmitida através dos tipos:

- paralinguagem ou sinais paralingüisticos, ou seja, os sons emitidos pelo aparelho fonador, mas que não fazem parte do sistema sonoro do idioma; por exemplo: "ah", "er", "uh", " $h m$ ", rir, gritar, pigarrear, bocejar, suspirar, entre outros;

- cinésica, ou seja, os movimentos do corpo, desde os gestos manuais, movimento dos membros, meneios de cabeça, até os mais sutis como as expressões faciais;

\footnotetext{
- Enfermelra Mestre em Enfermagem. Assistente do Departamento de Enfermagem Médico-Cirúrgica da Frscola de Infermagem da Universidado de săo Paulo.
} 
- proxêmica ou a distância mantida entre os participantes de uma interação;

- tacêsica, que se constitui no toque e todas as caracteristicas que o envolvem: pressão exercida, local onde se toca, idade e sexo dos comunicadores, entre outros;

uso do silêncio ou ausência das palavras que permitem interpretações diferentes dependendo do contexto social $e$ cultural em que ocorrem, pois podem ser impostas por normas sociais ou ser deliberados; podem, ainda, preceder uma tomada de decisão, o início ou o término da fala, ou mesmo uma conclusão a respeito da mensagem.

RECTOR \& TRINTA ${ }^{18}$ combinam quatro elementos para classificarem a forma de nos comunicarmos:

. a vacal-verbal: as palavras;

. a vocal-não verbal: os sinais paralingüísticos;

- a não vocal-verbal: as palavras escritas ou impressas;

- a não vocal-não verbal: expressões faciais, gestos, pasturas.

GUIRAUD ${ }^{9}$ estuda os sinais não-verbais corporais dividindo-os em dois grandes grupos:

- o formato do corpo, ou seja, a própria forma e aparência de um corpo que nos transmite informações sobre faixa etária, sexo, origem étnica e social, estado de saúde, entre outros;

- a relação do corpo com o ambiente e sua movimentação no espaço; aqui o autor inclui os elementos cinésicos, proxêmicos e paraverbais.

Todos os autores, porém, concordam que a comunicação não-verbal tem merecido pouca atenção dos pesquisadores, e que este desconhecimento pode dificultar as interações interpessoais por leitura incorreta do processo comunicativo. MONTAGU ${ }^{14}$ afirma que, devido a nossa progressiva sofisticação e falta de envolvimento reciproco, passamos a utilizar exagerađamente a comunicação verbal, chegando a, virtualmente, excluir de nossa experiência o universo da comunicação não-verbal, o que causa acentuado empobrecimentao da nossa comunicação.

O estudo do não-verbal pode resgatar a capacidade do enfermeiro de perceber com maior precisão os sentimentos do paciente, suas dúvidas e sua dificuldade de verbalização, bem como potencializar sua própria comunicaçåo (enquanto elemento transmissor de mensagens).

$\mathrm{Na}$ enfermagem, vários autores também afirmam que, apesar da palavra "comunicação" ser bastante usada, são poucos os enfermeiros que conhecem os tipos e formas de comunicação existentes, e a percebem como uma capacidade que pode e deve ser desenvolvida por esse profissional 1,20,22. 
Esse desconhecimento do enfermeiro é um grande problema de enfermagem, visto que a comunicação é considerada como instrumento básico na relação enfermeira-paciente, e aparece com frequiência nos livros e teorias de enfermagem como elemento fundamental nessa relação ${ }^{15,21,24 .}$

Aceitando-se a premissa de que, para ministrar atendimento de enfermagem adequado às necessidades do paciente, é preciso o enfermeiro compreendê-lo, e que esta compreensão se dá por meio do que é expresso por ele de maneira verbal e não-verbal 1,20 , é que o estudo do processo global da comunicação (verbal e não-verbal) precisa ser implementado.

Assim, acreditando na impossibilidade do paciente ou do enfermeiro deixar de se comunicar, já que qualquer comportamento, palavra ou mesmo silêncio, em um determinado contexto, tem valor de mensagem ${ }^{25}$, registramos algumas observações feitas durante consultas de enfermagem a pacientes adultos, em um hospital geral, sobre o toque, a distância mantida entre enfermeiro-paciente nas consultas e os objetos utilizados por ambos.

As observações contidas neste trabalho foram obtidas durante a fase de coleta de dados para a confecção da dissertação de Mestrado por nós apresentada à EEUSP ${ }^{20}$.

O referencial teórico utilizado para a análise dos dados foi $\mathrm{A}$. MONTAGU $^{14}$ e P. GUIRAUD ${ }^{9}$ na interpretação do toque; E. HALL ${ }^{10}$ na interpretação da distância interpessoal mantida entre enfermeiro e paciente; e, P. GUIRAUD ${ }^{9}$ e A. PEASE ${ }^{17}$ na compreensão dos objetos utilizados pelos enfermeiros e pacientes que pudessem definir ou delimitar a relação.

\section{OBJETIVOS}

Ciente da importância da compreensão da comunicação não-verbal, por serem os sinais, nesta utilizados, os que influem e definem as relações, além de permitirem a efetivação da comunicação do enfermeiro, é que realizamos este estudo com os objetivos de verificar:

- o momento e o local em que ocorre o toque enfermeiro e paciente nas consultas de enfermagem;

- a distância interpessoal mantida entre enfermeiro e paciente durante as interações;

. que objetos utilizados pelos pacientes e enfermeiros, definiram ou delimitaram a relação.

\section{METODO}

Utilizamos o método dedutivo para a realização de um estudo exploratório descritivo. 


\section{Campo de pesquisa e população}

Esta pesquisa foi realizada em um hospital governamental na cidade de São Paulo.

A população da pesquisa foi constituída pelos 13 enfermeiros das Clínicas Cirúrgica e Médica, que estavam cuidando de 13 pacientes adultos por ocasião da consulta de enfermagem no ambulatório. Este número representa o total de enfermeiros $(100 \%)$ que estavam atendendo pacientes adultos nas consultas de enfermagem, durante 0 periodo de coleta de dados, portanto observamos a interação entre 13 enfermeiros e 13 pacientes.

\section{Coleta de dados e procedimentos}

A coleta de dados foi realizada no período de dezembro de 1988 a junho de 1989, compreendendo, portanto, um período de 6 meses.

Todas as consultas de enfermagem foram observadas pela pesquisadora, que não interferia de modo direto na interação que estava ocorrendo entre enfermeiro-paciente.

Cada enfermeiro só foi observado uma vez nas consultas de enfermagem, já que estes dados fazem parte de uma pesquisa maior ${ }^{20}$, na qual a interação que ocorria entre a pesquisadora e o enfermeiro, alteraria os resultados finais de vários dados, se houvesse a observação de nova consulta de enfermagem com o mesmo enfermeiro.

\section{RESULTADOS E COMENTAARIOS}

Os resultados e comentários serão feitos primeiramente pela apresentação dos dados observados sobre a ocorrência do toque entre enfermeiro e paciente; segundo, pela distância mantida entre eles, durante as consultas e, em terceiro lugar, pela verbalização de interferências ocorridas devido ao uso de objetos.

Tocar é definido como a ação ou ato de sentir alguma coisa com a mão, apalpar, comover, sensibilizar, entre outros ?. O termo operacional é "sentir", e, embora o tato não seja em si uma emoção, seus elementos sensoriais induzem alterações neuronais, glandulares, musculares e mentais que, combinadas, denominamos emoção ${ }^{14}$.

Decorre deste fato que, muitas vezes, o tato não é "sentido" como uma sensação, e sim, efetivamente, como emoção. Usamos expressões que demonstram emoções com o toque: "tocado profundamente", "não me toques", "manter contato", entre outros.

MONTAGU 14 cita estudos feitos por enfermeiros que afirmam poder ser o uso do toque e da proximidade física a maneira mais importante de entrar em comunicação com um doente, e de demonstrar-lhe afeto, envolvimento, segurança e a noção de que é importante como ser humano. 
Nas consultas de enfermagem observadas e registradas pela autora, o toque entre enfermeiro e paciente ocorreu apenas nos momentos em que ambos se cumprimentaram (ao iniciar ou concluir a consulta de enfermagem) e nos momentos de execução dos procedimentos técnicos padronizados para cada tipo de cuidado e tratamento. Foram momentos em que, de certa forma, era "esperado" ou necessário que o toque ocorresse neste contexto de consulta: 1") porque assim era exigido tecnicamente (verificação de temperatura, curativo, troca de bolsa de colostomia, entre outros); $2^{\circ}$ ) porque culturalmente está incorporado e normatizado; apertar as mãos expressa, na nossa cultura, ausência de más intenções ou sinal de amistosidade.

Não houve nenhum outro momento nas consultas de enfermagem em que ocorresse 0 toque entre enfermeiro-paciente, apesar de culturalmente ser aceito que o profissional de enfermagem pode entrar em contato mais intimo com as pessoas, ou seja, pode tocar as pessoas que estão sob seus cuidados, mais freqüentemente que em outros tipos de interação.

Os enfermeiros tocaram os pacientes no local onde algum procedimento técnico estava sendo executado: no braço para verificação da PA e T; no abdome, para troca de curativo; na perna, para verificação de edemas, entre outros, e na mão direita para o cumprimento inicial e final nas consultas de enfermagem.

Como nenhum paciente solicitasse auxílio para deambular, subir na maca ou sentar-se, eles só foram tocados nas situações descritas anteriormente, e nos locais "esperados" (exigência técnica ou cultural).

$O$ toque, isoladamente, não foi usado como sinal que pudesse demonstrar qualquer tipo de afeição, integração ou mesmo como elemento regenerador, revitalizador.

GODOY ${ }^{8}$ refere que, pela ausência de publicações em língua portuguesa sobre o toque, poder-se-ia dizer que ele não é utilizado em nosso meio, na assistência de enfermagem; lembra, ainda, que por vários autores ele se configura como elemento curador (massagens, DO-IN, entre outros) $12,13,16$, e encoraja os enfermeiros a explorarem o toque como possibilidade de tratamento, comunicação e integração humana.

A disposição dos objetos na sala de consulta dificulta este contato mais próximo entre enfermeiro-paciente; existia uma mesa entre ambos durante toda a entrevista, e as cadeiras estavam dispostas frente à frente, com a mesa entre eles. Quando era o momento da execução dos procedimentos, ambos se dirigiam para a maca, onde o paciente permanecia deitado até o fim dos procedimentos técnicos. Vide desenho da sala de consulta em anexo (Anexo I).

Como foi dito anteriormente, o toque só ocorreu inserido em uma "sequiência técnica" a ser cumprida pelo enfermeiro; via de regra, o movimento para que o toque ocorresse foi iniciado pelo enfermeiro, e, segundo DAVI ${ }^{6}$ e STEINBERG ${ }^{23}$ as regras sociais determinam que 0 
iniciador do contato seja geralmente o mais velho ou o mais importante; daí podermos inferir que o enfermeiro assumiu o papel de dominante na relação, durante todas as consultas observadas.

KENNEDY; GARVIN 11 e LANE ${ }^{12}$ afirmam que tocar pode significar "status" e poder, pois é invadir o espaço pessoal das pessoas. Também pode ser considerado uma deferência (superior-subordinado) ou uma falta de deferência para com a outra pessoa (expôr o corpo do paciente na frente dos demais), dependendo do local e da maneira como ocorre o toque.

KENNEDY; GARVIN ${ }^{11}$ e MONTAGU ${ }^{14}$ relatam estudos mostrando que as mulheres são mais comumente tocadas e, também, tocam com maior freqüência as pessoas do que os homens. Porém, nas consultas de enfermagem observadas, não houve diferença em número de toques ocorridos entre pacientes, homens/mulheres, e enfermeiros, homens/mulheres.

Toda cultura e contexto têm um código tácito sobre a proximidade aceitável entre as pessoas, e a não observância das regras de distância pode pôr a perder uma tentativa de comunicação, pois a violação em torno do espaço de um individuo pode levar a interpretações errôneas ${ }^{10,23}$.

HALL 10 divide e caracteriza o espaço entre as pessoas em distância íntima (do contato a $40 \mathrm{~cm}$ entre as pessoas), pessoal (de $45 \mathrm{~cm}$ a $120 \mathrm{~cm}$ ), social (de $120 \mathrm{~cm}$ a $360 \mathrm{~cm}$ ) e pública (acima de $360 \mathrm{~cm}$ ), Afirma que, na distância intima, a presença do outro se impõe e pode tornar-se invasora pelo seu impacto sobre o sistema perceptivo do outro. O cheiro, o calor, o ritmo da respiração, o hálito do outro tornam-se bastante perceptíveis, portanto quando esta distância ocorre, sem que a pessoa envolvida queira tornar-se íntima da outra, ela mantém-se tão imóvel quanto possivel, com os músculos contraídos e os olhos voltados para o infinito ou se afasta do outro.

COOPER ${ }^{3}$ cita estudos mostrando que cada paciente tem um espaço social ao redor de si, visto como extensão de seu próprio corpo. O tamanho deste espaço pessoal varia de um para outro, porém podemos ter uma idéia sobre ele observando onde o paciente coloca seu roupão, seus chinelos, seu livro, etc. Se o enfermeiro ignora estes sinais e não respeita esta zona, ou não pede licença para invadi-la quando necessário, obtém resultados diferentes na sua interação e na assistência prestada.

$\mathrm{Na}$ sua tese de doutorado, CARVALHO ${ }^{2}$ concluiu ser a idade a única variável que influi na extensão do território desejado pelos internados: pacientes de idade entre 18 e 30 anos têm necessidade de maior espaço pessoal que os pacientes na faixa de idade entre 42 a 60 anos.

Nas consultas de enfermagem observadas foi mantida uma distância social (aproximadamente $130 \mathrm{~cm}$ ) enquanto as perguntas eram feitas, e uma distância íntima (do toque aos $40 \mathrm{~cm}$ ), na realização dos 
procedimentos. Em 5 consultas de enfermagem foi observado 0 "imobilismo" do paciente, a contração de seus músculos e os olhos voltados para 0 teto da sala, enquanto ele estava sendo examinado pelo enfermeiro. Os enfermeiros, ao final das consultas, não referiram perceber este comportamento nos pacientes.

PEASE 17 afirma que situar-se estrategicamente com relação às demais pessoas é um modo efetivo de obter sua colaboração. Sentar-se em frente a uma pessoa, com uma mesa no meio, cria um ambiente competitivo e pode significar que se está na defensiva; esta situação pode levar as duas pessoas a reafirmar seus pontos de vista, já que a mesa é uma barreira sólida entre ambos; também pode estabelecer uma situação de superior - subordinado quando a entrevista é realizada.

A relação de poder e diferença de papéis enfermeiro-paciente fica clara quando o enfermeiro senta-se atrás da mesa, ou permanece de pé ao lado da maca, com o paciente "deitado" (mais passivo) e mais exposto fisicamente à visão e ao toque do enfermeiro ${ }^{10,17}$.

Se o objetivo de alguém é entender o ponto de vista da outra pessoa, para fazer com que se sinta tranquilo na. relação com ele, a posição competitiva (com uma mesa entre os dois) não cumpre esta finalidade ${ }^{17}$. Isto nos leva a pensar se os enfermeiros não precisam rever, também a posição dos móveis nos consultórios de enfermagem, como forma de facilitar sua relação com o paciente 20 : ou se, conhecendo estes sinais, o enfermeiro não precisa adotar outra posição durante alguns momentos das consultas de enfermagem.

Com relação à categoria de sinais "objetos" utilizados pelos pacientes e enfermeiros, que definiram ou delimitaram a relação, podemos apontar o uso de roupa branca pelos enfermeiros, enquanto o paciente estava com roupas sociais/esportivas, deixando claro quem era o profissional na interação. Também o uso do crachá identificativo dos enfermeiros e, muitas vezes, o fato de pacientes segurarem na mão o cartão de identificação, para consulta no hospital, definiam os papéis na interação.

O enfermeiro foi sempre o elemento mais ativo na relação, pois foi quem manipulou os equipamentos e materiais de consumo usados nas técnicas executadas, passando a informação que o conhecimento estava sendo dado por ele. Ao paciente coube o papel de elemento mais passivo no seu cuidado, e pouco indagador às condutas adotadas para a sua própria recuperação.

\section{CONCLUSŌES}

Diante da análise dos resultados encontrados após a observação das interações entre enfermeiro e paciente nas consultas de enfermagem podemos concluir que:

- o toque entre enfermeiro e paciente ocorreu somente nas máos ao cumprimentarem-se no início e no término das consultas, e nos 
locais que exigiam algum procedimento técnico durante as consultas (troca de curativo, verificação da T e PA, entre outros), durante a execução dos procedimentos pertinentes;

- a distância mantida entre enfermeiro e paciente foi uma distância social, enquanto durava a entrevista, e uma distância íntima, enquanto eram executados os procedimentos;

- os objetos que usados, definiram ou delimitaram a relação, foram: da parte do enfermeiro, a roupa branca, o uso do crachá e a manipulação dos equipamentos e materiais de consumo; e da parte do paciente, o seu cartão de identificação.

Finalizando, podemos dizer que é urgente a necessidade dos enfermeiros conhecerem, ou resgatarem a nivel consciente, a comunicação não verbal emitida por eles e pelos pacientes, como maneira de entender melhor o que acontece nas relações entre enfermeiro-paciente. A comunicação não verbal é uma verdadeira "delatora" das nossas intenções e objetivos, assim como das nossas crenças e sentimentos. $O$ paciente, por estar correndo riscos, está muitas vezes com sua percepção aguçada. Ele está atento às nossas mensagens.

O que nós estamos transmitindo à população?

O que estamos percebendo da comunicação não verbal dos nossos pacientes?

SILVA, M.J.P. da The touch and the interpersonal distance during adults nursing consultation. Rev. Esc. Enf. USP, v. 25, n. 3, p. 309-18, Dec. 1991.

The present article reports on the observation made during adults nursing consultation in $a$ hospttal of the of City São Paulo: concerning about time and place where nuise and patient contacted each other during the consultation; the interpersonal distance maintained between then during all time; and take comments about the meaning of nouverual signs in interpersonal interaction. It discusses the objects used by nurses and pattents whach were useful for identification or definition of the maintained relationsmip.

UNITERMS: Touch. Interpersonal distance. Nonverbal communication.

\section{REFERENCIAS BIBLIOGRAFICAS}

01. BLONDIS, M.N.; JACKSON, B.E. Nonverbal communication with pationts: back to the human touch. New York, John Wiley, 1982. cap. 1, p. 2-29: What is nonverbal communication?

02. CARVALHO, D.V. Necessidade territorial do paciente hospitalizado. Såo Paulo, 1987. 115 p. Tese (Doutorado) - Escola de Enfermagem, Universidade de sta Paulo.

03. COOPER, J. Actions really do speak louder than words. Nursing, v. 9, n. 4, p. 29-32, 1979.

04. CORRAZE, J. As comunicacões não verbais. Rlo de Janeiro, Zahar, 1982.

05. DAS, D.M.H. Forms of communication. Nurs. J. India., v. 66, n. 3, p. 65-7, 1975. 
06. DAVIS, F. Comaniesegro nto verbal. Sto Paulo, Summus, 1979.

07. FLRRREIRA, A.B. de H. Novo dielonário da lígua portuguesa. 2 ed. Rio de Janeiro, Nove Fronteirs, 1986.

08. GODOY, A.N: O toque como elemento de integraça ne relaçăo enfermeiro-cliente. Rov. Gatichs do Enf., v. 6, n. 2, p. 305-16, 1985.

09. GUIRAUD, P. El lenguage del cuerpo. México, Fondo de Cultura Económica, 1986.

10. HALI, F.T. A dimenotio culta. Lisboa, Relógio D'Agua, 1986.

11. KENEDDY, C.W.; GARVIN, B.J. The effect of status and gender on interpersonal relationships in nursing. Nurs. Forum, v. 20, n. 3, p. 275-85, 1981.

12. LANE, P.L. Nurse-client perceptions: the double standard of touch. Issues Mental Foulth Nart., v. 10, n. 1, p. 1-13, 1989.

13. MENTGEN, J.L. Therapeutic touch: a healing art. J. of Assoc. Pediat. Oncology Nurses, v. 6, n. 2, p. 29-30, 1989.

14. MONTAGU, A. Tocar - o significado humano da pale. Săo Paulo, Summus, 1988.

15. ORLANDO, I.J. Tho dinamio nurse-patient relationship: function, procees and principles. New York, Putnam's, 1961.

16. PDARCE, J. The power of touch. Nurs. Times, v. 84, n. 24, p. 26-8, 1988.

17. PEASE, A. El lenguage del cuerpo. Barcelona, Paidós, 1989.

18. RECTOR, M.; TRINTA, A.R. Comunicagĩo nĩo verbal: a gestualidade brasileira. Petropolis, Vozes, 1986.

19. Santamlla, L. o que é cemítica. Săo Paulo, Brasiliense, 1987.

20. SILVA, M.J.P. da A percepç̃o das onfermeiras sobre a comunicacão não verbal dos pacientex. S4̌o Paulo, 1989. 113 p. Dissertaç̆o (Mestrado) - Escola de Enfermagem, Universidade de sto Paulo.

21. STBRANBLLI, M.C. Comunicagăo em enfermagem: ejargăo profiasional ou realidades? Bol. Int. ETUSP, v. 4, n. 6, p. 2-5, 1988.

22. STEFANELLI, M.C. Ensino de tecnicas de comunicacio terapética onfermoira-pacionto. Sao Paulo, 1985.' 163 p. Tese (Doutorado) - Fscola de enfermagem, Universidaite. de sto Paulo.

23. STRINBFrG, M. Os elementos nǐo verbais da comnnicaç̃o. Săo Paulo, Atual, 1988.

24. SUNDFHEN, S.J. et al. Nurwe-alient interaction: implementing the nursing process. Saint Louis, Mosby, 1876. cap. 4, p. 68-107: Communication.

25. WATZLAWICK, P. et al. Pragmitice de comunicacio humana. São Paulo, Cultrix, 1967. cap. 2. p. 44-64: Alguns axjomas conjeturais de comunicacto.

Recebido em $27-9-80$

Aprovado em 2-5-91 
ANEXO I

SALA DE CONSULTA DE ENFERMAGEM

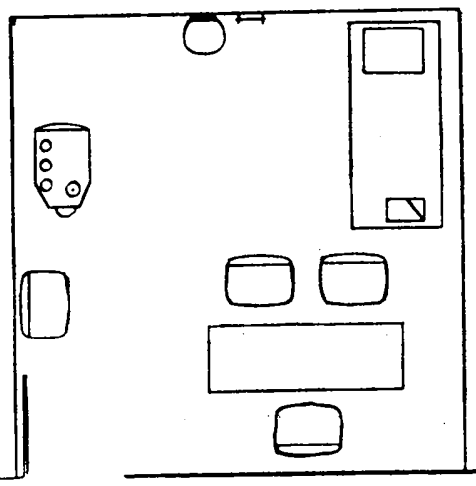

corredor de circulação 